\title{
Impact of Physician-Pharmacist Collaborative Anticoagulation Clinic on Warfarin Therapy Management
}

Binaya Sapkota $^{1^{*}}$, Sweta Shrestha ${ }^{2}$, Anurodh Ghimirey ${ }^{3}$, Navin Chandra Gautam $^{4}$ and Rajani Shakya ${ }^{2}$

${ }^{1}$ Government of Nepal Civil Service Hospital, Minbhawan, Kathmandu, Nepal

${ }^{2}$ Kathmandu University School of Science, Dhulikhel, Kavre, Nepal

${ }^{3}$ Karnali College of Health Sciences, Kathmandu, Nepal

${ }^{4}$ Department of Cardiovascular Surgery, Shahid Gangalal National Heart Center, Bansbari, Kathmandu, Nepal

*Corresponding author: Binaya Sapkota, Government of Nepal Civil Service Hospital, Minbhawan, Kathmandu, Nepal, Tel: +977-9851134925; Fax: +977-014107003; E-mail: sapkota.binaya@gmail.com

Received date: Jul 01, 2014, Accepted date: Mar 05, 2015, Publication date: Mar 09, 2015

Copyright: (c) 2015 Sapkota B, et al. This is an open-access article distributed under the terms of the Creative Commons Attribution License, which permits unrestricted use, distribution, and reproduction in any medium, provided the original author and source are credited.

\begin{abstract}
Background: Approximately $40-45 \%$ interpatient variability in response to warfarin is still conspicuous. These uncertainties contribute to warfarin's underuse in patients who could benefit from anticoagulation. Anticoagulation clinics provide systematic method for anticoagulation management, warfarin dosage adjustment and patient education.

Methods: Prospective case-control study was conducted from May to September 2012 at surgical outpatient department at Shahid Gangalal National Heart Center, Nepal to compare physician-pharmacist collaborative anticoagulation clinic (PPAC) approach on warfarin therapy management (case) to usual anticoagulation therapy (UAT) provided by physicians (control). Control $(n=75)$ and case group $(n=75)$ were selected by systematic random sampling in such a way that ratio of control to case is $1: 1$. Participants who required warfarin for their mechanical heart valve replacement for at least three months via UAT approach were included. They were counseled on dosage regimen, diet, signs and symptoms of hemorrhagic or thromboembolic events. Patient anticoagulation therapy leaflet was provided to case group. Data related to warfarin were analyzed using repeated measure ANOVA. A $p<0.05$ was considered statistically significant.
\end{abstract}

Results: Baseline international normalized ratio (INR) value in UAT approach $(4.45 \pm 2.00)$ was changed to 4.21 $\pm 1.75,4.45 \pm 2.00$ and $4.21 \pm 1.75$ with $p 0.807,0.000$ and 0.807 during first, second and third follow ups. In PPAC, baseline INR value $(0.99 \pm 0.81)$ was constantly maintained to $2.21 \pm 0.77$ with $p 0.000$ throughout all follow ups. In PPAC, baseline monthly cost minimizations were statistically significant, with $p 0.000$ on each.

Conclusion: INR value of patient in PPAC approach was statistically within recommended range (p 0.000 on each condition). The study showed that INR value of the patients can be kept within the recommended range when physicians and pharmacist collaboratively manage patients' warfarin therapy.

Keywords: Anticoagulation clinic; International normalized ratio; Warfarin

\section{Introduction}

The factors that affect patients' response to warfarin therapy are not completely elucidated despite more than 50 years of its use in medical practice. Approximately 40-45 per cent observed interpatient variability in response to warfarin is still conspicuous. These uncertainties in response contribute to warfarin's underuse in some patients who could benefit from anticoagulation. Dietary factors and concomitant medicines use may alter pharmacokinetics of warfarin. This makes maintaining international normalized ratio (INR) within narrow therapeutic range and maintaining stability of INR problematic [1]. Strictly maintaining the INR within the therapeutic range is required to ensure treatment efficacy with the lowest possible risks of thromboembolic and bleeding complications [2]. The American College of Chest Physicians (ACCP) guidelines recommend daily monitoring of patients on warfarin therapy until their INR is within therapeutic range for at least two consecutive days. Patients are monitored two to three times per week for the next one to two weeks and then weekly until their INR levels are stable within the therapeutic range. Once they have achieved this anticoagulation control, patients are monitored monthly [3].

Gadisseur et al. concluded that anticoagulation should be monitored by specialized anticoagulation clinic (ACs) to minimize hemorrhagic and thromboembolic risks. 2 The ACCP Consensus Conference on Antithrombotic Therapy also endorsed ACs and concluded that failure to utilize them increases the risk of legal liability [4].

The ACs have been established in the Netherlands, USA, UK and other countries to improve anticoagulation control in warfarin-treated patients. The physician-pharmacist collaborative anticoagulation clinics (PPAC) may have lower-range anticoagulation, more time within the stipulated range of INR, lower risk of significant bleeding, lower rates of thromboembolic events and reduction of annual health care costs [1]. However, there is no such AC in Nepal till date. In 
Nepal, physicians manage warfarin therapy without any involvement of pharmacist in the anticoagulation-related decision making process. This study has been carried out to strengthen the physicianpharmacist collaboration to manage warfarin therapy to achieve better therapeutic outcome with minimum adverse effects. This very first research of its kind in Nepal emphasizes the need for anticoagulation clinic in the country.

\section{Methods}

\section{Study site and anticoagulation clinic}

The study was carried out at 450-bed Shahid Gangalal National Heart Center (SGNHC), Bansbari, Kathmandu. It is one of the largest semi-autonomous tertiary care heart centers in Nepal serving 100,000 outpatients per year.5 Only the anticoagulation physicians used to counsel and provide anticoagulation related services to the patients prior to this study. The involvement of physician into anticoagulation service management comprised the PPAC approach. The PPAC was stationed on the surgical outpatient unit of the hospital. The clinic was managed by seven cardiac surgeons and one pharmacist. The AC Pharmacist provided patient care services such as patient education, dosage adjustments, screening for drug-drug, drug-food, drug-disease interactions and INR monitoring. Pharmacist utilized the approved protocols for warfarin monitoring and dosage adjustments to obtain maximal benefit with warfarin therapy. Pharmacist evaluated patients during an initial clinic visit; all subsequent follow-up contacts were made via telephone. Patients were dismissed from the AC if they repeatedly failed to keep appointments with the AC and the laboratory appointments for INR monitoring.

\section{Ethical approval}

The ethical permission to carry out the research was taken from the ethical review board in SGNHC. The patients were informed about the research and verbal consent was received from all the patients who participated in the research. They were assured of their confidentiality and anonymity and also assured that data would be used solely for research purpose.

\section{Study design, selection of controls and cases}

The prospective, case-control study was conducted from May to September 2012 to compare the warfarin therapy management in PPAC to usual anticoagulation therapy (UAT) approach. Prospective patients who required anticoagulation therapy for their thromboembolic and hemorrhagic events came to the clinic. They were categorized into UAT patient group (control group) and PPAC patient group (case group) by systematic random sampling. Controls were subjected to physician-only intervention. They were similar to the cases except for the absence of pharmacist intervention.
Pharmacist only collected data from them for comparing them with the cases. Cases were taken from the same AC. The effects in the warfarin therapy management on the controls were compared with that on the cases.

\section{Inclusion criteria}

All the patients' $\geq 18$ years of age who were receiving warfarin therapy for at least three months via UAT were included in the study. Warfarin therapy duration of a minimum of two months allowed the researcher sufficient time to evaluate warfarin usage after the initial dosage titration period. During this time period, there were no changes in any of the AC personnel. Three times follow up data were taken from the patients within three months follow up period on monthly basis.

\section{Exclusion criteria}

The INR values of the patients were excluded if they were within the first 30 days of taking warfarin. Patients with contraindications to warfarin therapy were identified during the initial period and were excluded from the study.

\section{Research tools, sample size, data collection and statistical analysis}

Participation in an AC should have resulted in minimum 10 percent improvement in anticoagulation control [5,6]. Thus, with power equal to 0.9 (i.e. $\beta=0.1$ ), prevalence in the control group equal to 0.15 and odds ratio to be detected 3.5, sample size of at least 150 patients (75 in each model) was taken. The study was two-tailed with level of significance ( $\alpha) 0.05$ [7]. The details of patients' demographics as well as warfarin therapy were entered in the statistical package for social sciences (SPSS) software version 17. All data were collected by the pharmacist in collaboration with the physician from May to September 2012. Clinical and economic outcomes were compared between UAT and PPAC patients. The costs of warfarin therapy were used to assess the financial impact of the PPAC. Data related to warfarin therapy were analyzed using repeated measure analysis of variance (ANOVA). A $\mathrm{p}<0.05$ was considered statistically significant i.e. confidence interval equal to $95 \%$.

\section{Results}

In the UAT approach, 37.3 and 32 percent patients in the age group 20-29 years and 30-39 years came to the clinic whereas in the PPAC approach, the distribution was 32 and 22.7 percent patients in the same age group. In the UAT approach, 61.3 percents females came for their valve related complications and the figure was nearly same in the PPAC approach (65.3\%) (Table 1).

\begin{tabular}{|l|l|l|l|l|}
\hline Variables & \multicolumn{2}{|l|}{ UAT $^{1}$ Approach } & \multicolumn{2}{l|}{ 2PPAC Approach } \\
\hline Age (in years) & Frequency & Percentage & Frequency & Percentage \\
\hline $\mathbf{1 0 - 1 9}$ & 4 & 5.3 & 8 & 10.7 \\
\hline $\mathbf{2 0 - 2 9}$ & 28 & 37.3 & 24 & 32.0 \\
\hline $\mathbf{3 0 - 3 9}$ & 24 & 32.0 & 17 & 22.7 \\
\hline
\end{tabular}


Citation: Sapkota B, Shrestha S, Ghimirey A, Gautam NC, Shakya R (2015) Impact of Physician-Pharmacist Collaborative Anticoagulation Clinic on Warfarin Therapy Management. J Blood Disorders Transf 6: 256. doi:10.4172/2155-9864.1000256

Page 3 of 5

\begin{tabular}{|c|c|c|c|c|}
\hline $40-49$ & 10 & 13.3 & 16 & 21.3 \\
\hline 50-59 & 6 & 8.0 & 8 & 10.7 \\
\hline $60-69$ & 3 & 4.0 & 1 & 1.3 \\
\hline $70-79$ & 0 & 0 & 1 & 1.3 \\
\hline \multicolumn{5}{|c|}{ Gender of patients } \\
\hline Male & 29 & 38.7 & 26 & 34.7 \\
\hline Female & 46 & 61.3 & 49 & 65.3 \\
\hline
\end{tabular}

Table 1: Basic characteristics of study population $(\mathrm{n}=150$ : case $=75$; control=75) (Mean age in UAT=33.57 \pm 11.85 years; mean age in $\mathrm{PPAC}=34.73 \pm 13.23$ years).

In the UAT approach, 29.3 percent patients came to the clinic for mitral valve replacement with sinus rhythm (MVR with SR). This was followed by patients requiring double valve replacement (DVR) (26.7\%) and mitral valve replacement with atrial fibrillation (MVR with AF) (25.3\%). In the PPAC approach also, MVR with SR cases were handled more (36\%) compared to DVR (29.3\%) but the aortic valve replacement (AVR) cases took the third position with 21.3 percent cases (Table 2).

\begin{tabular}{|l|l|l|l|l|}
\hline \multirow{2}{*}{ Indication of Warfarin therapy } & UAT Approach & \multicolumn{2}{l|}{ PPAC Approach } \\
\cline { 2 - 6 } & Frequency & Percentage & Frequency & Percentage \\
\hline Aortic valve replacement & 14 & 18.7 & 16 & 21.3 \\
\hline Mitral valve replacement with sinus rhythm & 22 & 29.3 & 27 & 36.0 \\
\hline Mitral valve replacement with atrial fibrillation & 19 & 25.3 & 10 & 13.3 \\
\hline Double valve replacement & 20 & 26.7 & 22 & 29.3 \\
\hline
\end{tabular}

Table 2: Warfarin therapy related characteristics of patients.

The INR value of the patient enrolled on the UAT approach was changed from the baseline value $4.45 \pm 2.00$ to $4.21 \pm 1.75$ after first follow up and the change was not statistically within the recommended range $(\mathrm{p}=0.807)$. INR value remained nearly constant $(4.45 \pm 2.00)$ and was statistically within the recommended range after second follow up $(\mathrm{p}=0.000)$. In the PPAC group, the within subject variations on the baseline INR value after first, second and third follow ups were statistically significant $(\mathrm{p}=0.000$ on each condition). Similarly, the variations after first and second follow up as well as after first and third follow up were also significant $(\mathrm{p}=0.000$ on each condition) (Table 3).

\begin{tabular}{|l|l|l|}
\hline Category 1 (INR within range) & Category 2 (INR within range) & P value \\
\hline UAT Approach & After first follow up (4.21 \pm 1.75$)$ & 0.807 \\
\hline Baseline $(4.45 \pm 2.00)$ & After second follow up $(4.45 \pm 2.00)$ & 0 \\
\hline & After third follow up (4.21 \pm 1.75$)$ & 0.807 \\
\hline After first follow up & After second follow up & 0.807 \\
\hline & After third follow up & 0 \\
\hline After second follow up & After third follow up & 0.807 \\
\hline PPAC Approach & & \\
\hline Baseline (0.99 \pm 0.81$)$ & After first follow up $(2.21 \pm 0.77)$ & 0 \\
\hline
\end{tabular}


Citation: Sapkota B, Shrestha S, Ghimirey A, Gautam NC, Shakya R (2015) Impact of Physician-Pharmacist Collaborative Anticoagulation Clinic on Warfarin Therapy Management. J Blood Disorders Transf 6: 256. doi:10.4172/2155-9864.1000256

Page 4 of 5

\begin{tabular}{|l|l|l|}
\hline & After second follow up $(2.21 \pm 0.77)$ & 0 \\
\hline & After third follow up $(2.21 \pm 0.77)$ & 0 \\
\hline & After second follow up & 0 \\
\hline After second follow up & After third follow up & 0 \\
\hline
\end{tabular}

Table 3: Comparison of INR values within recommended range between UAT and PPAC approaches.

The baseline total monthly cost of warfarin therapy in the UAT approach was changed from $\$ 1.23 \pm 0.50$ to $\$ 1.19 \pm 0.55, \$ 1.23 \pm 0.54$ and $\$ 1.25 \pm 0.53$ after first, second and third follow ups respectively and these were statistically not significant, with $\mathrm{p}=1.000$ on each condition. Similarly, the total monthly cost minimization after first and second follow up was also not significant $(\mathrm{p}=0.079)$ whereas the cost minimization after first and third follow up was statistically significant $(\mathrm{p}=0.043)$. In the PPAC approach, the baseline total monthly cost of warfarin therapy was changed from $\$ 1.13 \pm 0.44$ to $\$ 0.65 \pm 0.22, \$ 0.66 \pm 0.21$ and $\$ 0.66 \pm 0.21$ respectively and these cost minimizations were statistically significant, with $\mathrm{p}=0.000$ on each condition. However, the cost minimizations after first and second follow up as well as after first and third follow up were not statistically significant, with p 1.000 on each condition (Table 4).

\begin{tabular}{|c|c|c|}
\hline Category 1 (Total monthly cost of Warfarin therapy) & Category 2 (Total monthly cost of Warfarin therapy) & $P$ value \\
\hline \multicolumn{3}{|l|}{ UAT Approach } \\
\hline \multirow[t]{3}{*}{ Baseline (mean cost: $\$ 1.23 \pm 0.50$ ) } & After first follow up (mean cost: $\$ 1.19 \pm 0.55$ ) & 1.000 \\
\hline & After second follow up (mean cost: $\$ 1.23 \pm 0.54$ ) & 1.000 \\
\hline & After third follow up (mean cost: $\$ 1.25 \pm 0.53$ ) & 1.000 \\
\hline \multirow[t]{2}{*}{ After first follow up } & After second follow up & 0.079 \\
\hline & After third follow up & 0.043 \\
\hline After second follow up & After third follow up & 1.000 \\
\hline \multicolumn{3}{|l|}{ PPAC Approach } \\
\hline \multirow[t]{3}{*}{ Baseline (mean cost: $\$ 1.13 \pm 0.44$ ) } & After first follow up (mean cost: $\$ 0.65 \pm 0.22$ ) & 0.000 \\
\hline & After second follow up (mean cost: $\$ 0.66 \pm 0.21$ ) & 0.000 \\
\hline & After third follow up (mean cost: $\$ 0.66 \pm 0.21$ ) & 0.000 \\
\hline \multirow[t]{2}{*}{ After first follow up } & After second follow up & 1.000 \\
\hline & After third follow up & 1.000 \\
\hline After second follow up & After third follow up & 1.000 \\
\hline
\end{tabular}

Table 4: Comparison of total monthly cost of warfarin therapy between UAT and PPAC approaches.

\section{Discussion}

In this research, all the participants required warfarin for their mechanical heart valve replacement to prevent blood clotting on the valve wall. Chiquette et al. reported 23 percent participants required warfarin on UMC group and 14 percent on AC group for their mechanical heart valve replacement [8]. Bungard et al. reported 24 percent participants required warfarin for their mechanical heart valve replacement [9]. The difference among these researches and the present research is on the approach and target patients of the clinic. In all of these researches, the authors analyzed the patients medication history of the patients on long-term warfarin therapy and the registry was from medical unit whereas in the present study the authors collected data prospectively from the surgical outpatient unit.
The INR value of the patient enrolled in the UAT approach was not statistically within the recommended range after first follow up $(p=0.807)$. Patients might not have been much aware of the importance of INR value within recommended range. In the PPAC group, the within subject variations throughout first, second and third follow ups after the baseline condition were statistically significant ( $p$ 0.000 on each condition). Young et al. also concluded that 73.4 percent patients enrolled on the pharmacist managed anticoagulation clinic (PC) group had their INR values within targeted therapeutic range (TTR) whereas the corresponding percentage on the usual care (UC) group was 64.8. In the research by Chiquette et al., 64.6 percents patients enrolled on the UMC group found their INR within targeted range whereas the corresponding frequency was 82.7 percents on the AC group [8]. 
Citation: Sapkota B, Shrestha S, Ghimirey A, Gautam NC, Shakya R (2015) Impact of Physician-Pharmacist Collaborative Anticoagulation Clinic on Warfarin Therapy Management. J Blood Disorders Transf 6: 256. doi:10.4172/2155-9864.1000256

Page 5 of 5

The baseline total monthly cost of warfarin therapy in the UAT approach changed non-significantly after first, second and third follow ups, with $p 1.000$ on each condition. The total monthly cost minimization after first and second follow up was also not significant $(\mathrm{p}=0.079)$. In the PPAC approach, the baseline cost minimizations of warfarin therapy were statistically significant, with $p=0.000$ on each condition. The cost minimization observed in the PPAC approach might be the result of the decreased warfarin dose.

\section{Conclusions}

INR value of patient in PPAC approach was statistically within recommended range ( $\mathrm{p}=0.000$ on each condition). The baseline cost minimizations of warfarin therapy were statistically significant, with $\mathrm{p}=0.000$ on each condition in the PPAC approach. Hence, the study showed that INR value of the patients can be kept within the recommended range when physicians and pharmacist collaboratively manage patients' warfarin therapy. This will also reduce the total monthly cost of warfarin therapy.

\section{Acknowledgements}

The authors would like to acknowledge faculties from Kathmandu University School of Science for their valuable contribution in conducting this research. The authors acknowledge Shahid Gangalal National Heart Center ethics committee for the ethical permission to carry out the research and all the patients who gave consent to participate in the research.

\section{Authors' Contributions}

BS was the anticoagulation pharmacist, involved in conception, design of the study, data collection, analysis, manuscript writing and submission; SS contributed in literature review and data analysis; AG contributed in literature review and manuscript writing; NCG was the anticoagulation physician, involved in conception and design of study, contributed in data collection and manuscript writing; RS contributed in conception and design of study and manuscript writing.

\section{References}

1. Young S, Bishop L, Twells L, Dillon C, Hawboldt J, et al. (2011) Comparison of pharmacist managed anticoagulation with usual medical care in a family medicine clinic. BMC Fam Pract 12: 88 .

2. Gadisseur APA, Breukink-Engbers WGM, van der Meer FJM, van den Besselaar AMH, Sturk A, et al. (2003) Comparison of the Quality of Oral Anticoagulant Therapy Through Patient Self-management and Management by Specialized Anticoagulation Clinics in the Netherlands: A Randomized Clinical Trial. Arch Intern Med 163: 2639-2646.

3. Chamberlain MA, Sageser NA, Ruiz D (2001) Comparison of anticoagulation clinic patient outcomes with outcomes from traditional care in a family medicine clinic. J Am Board Fam Pract 14: 16-21.

4. Hirsh J, Dalen JE, Deykin D, Poller L, Bussey H (1995) Oral anticoagulants. Mechanism of action, clinical effectiveness, and optimal therapeutic range. Chest 108: 231S-246S.

5. http://www.sgnhc.org.np/anual_report_2007/full_book.pdf.

6. Wilson SJ, Wells PS, Kovacs MJ, Lewis GM, Martin J, et al. (2003) Comparing the quality of oral anticoagulant management by anticoagulation clinics and by family physicians: a randomized controlled trial. CMAJ 169: 293-298.

7. Strom BL, Kimmel SE (2006) Textbook of Pharmacoepidemiology. John Wiley and Sons Ltd, UK: 463-470.

8. Chiquette E, Amato MG, Bussey HI (1998) Comparison of an anticoagulation clinic with usual medical care: anticoagulation control, patient outcomes, and health care costs. Arch Intern Med 158: 1641-1647.

9. Bungard TJ, Gardner L, Archer SL, Hamilton P, Ritchie B, et al. (2009) Evaluation of a pharmacist-managed anticoagulation clinic: Improving patient care. Open Med 3: e16-21. 